Universidad de Lima

Facultad de Psicología

Carrera de Psicología

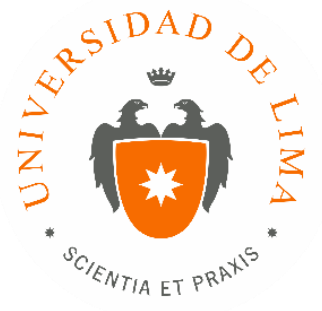

\title{
EXPERIENCIA DE TRABAJO CON MENORES INSTITUCIONALIZADOS EN UN CENTRO DE ATENCIÓN RESIDENCIAL DE LIMA
}

Trabajo de suficiencia profesional para optar el título profesional de Licenciado en Psicología Jorge Enrique Sifuentes Palomino

Código 20101887

Lima - Perú

Febrero de 2019 


\section{EXPERIENCIA DE TRABAJO CON MENORES INSTITUCIONALIZADOS EN UN CENTRO DE ATENCIÓN RESIDENCIAL DE LIMA}




\section{TABLA DE CONTENIDO}

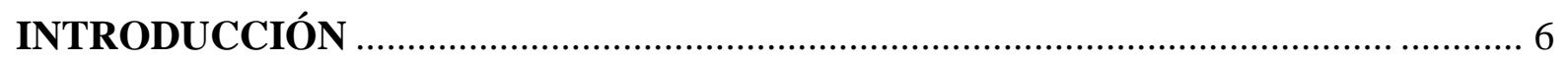

CAPÍTULO I: IDENTIFICACIÓN DEL PROBLEMA .................................................

CAPÍTULO II: DESCRIPCIÓN DE LAS ACTIVIDADES REALIZADAS ...............11

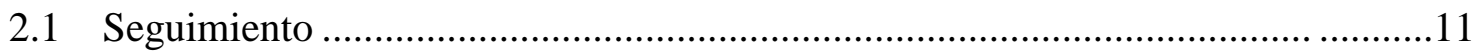

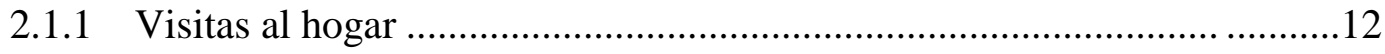

2.1.2 Visitas a eventos deportivos.........................................................12

2.1.3 Seguimiento en horarios de alimentación .............................................12

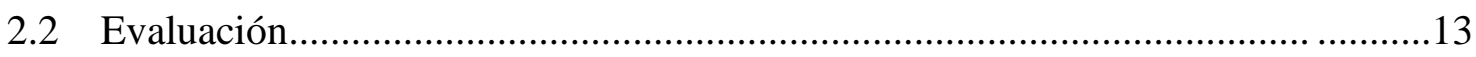

2.2.1 Aplicación de pruebas .......................................................................13

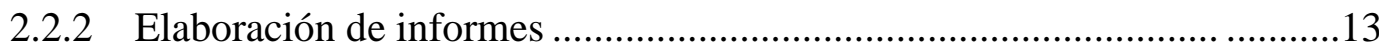

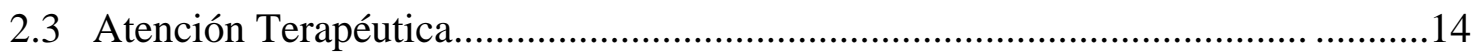

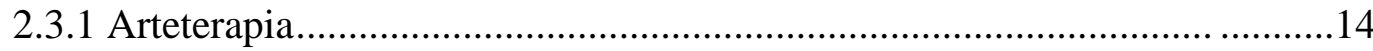

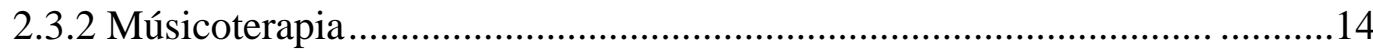

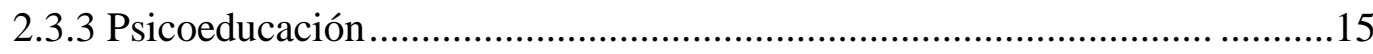

2.4 Elaboración de charlas para auxiliares .......................................................... 15

CAPÍTULO III: RESULTADOS DE LA INTERVENCIÓN ......................................16

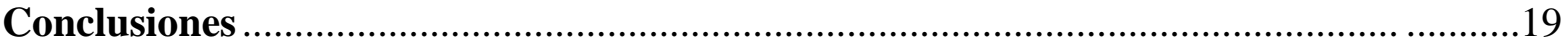

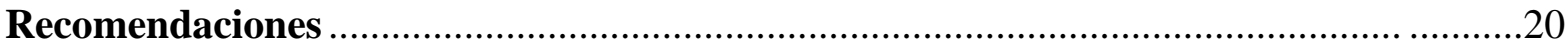

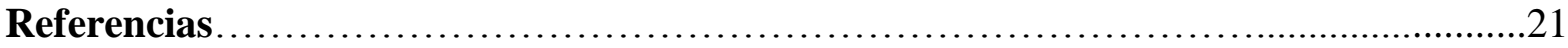

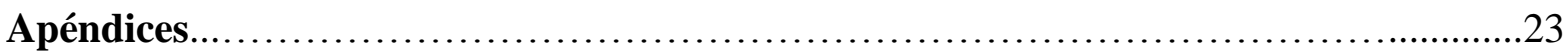




\section{ÍNDICE DE APÉNDICES}

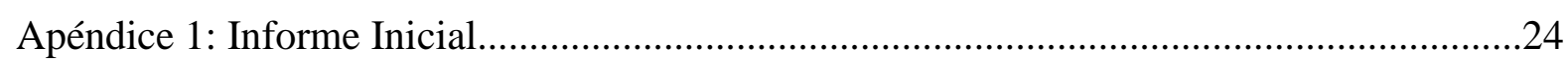

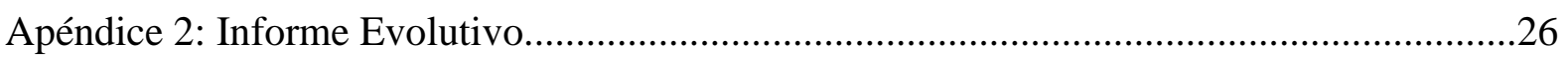

Apéndice 3: Cuadro de puntajes de evaluación del área emocional......................................28

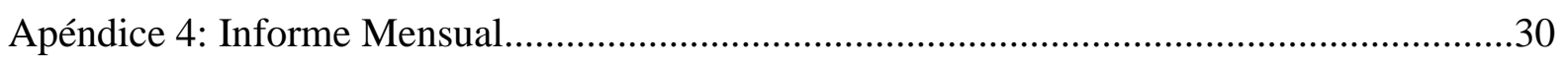

Apéndice 5: Guía de Actividades de Charla de Orientación...................................................33 


\section{INTRODUCCIÓN}

El abandono de menores es un fenómeno social que se suscita en la actualidad en todas las sociedades. Este problema llama la atención por que se sigue presentando en las familias, de forma recurrente, y con mayor incidencia en familias de escasos recursos. Existen factores como la violencia familiar, el maltrato infantil, el consumo de drogas, la pobreza y otros aspectos que impactan de forma negativa en este problema y que terminan siendo las causas de este fenómeno.

Los efectos de ser víctimas de algún tipo de maltrato y abandono durante la infancia son variados, siendo los más evidentes el retraimiento, el comportamiento agresivo, y las dificultades para poder prestar atención y concentrarse (Fernández, 2014). Este tipo de dificultades son las que enfrentan muchos menores que han sido víctimas de este problema.

El presente trabajo describe la experiencia de trabajo desde la perspectiva de interno de Psicología, en una institución que alberga menores de edad que han sido víctimas de abandono y que requieren residencia, alimentación, vestimenta, educación, así como reinsertarse a la sociedad de forma adecuada.

El objetivo de este reporte es describir el contexto en el cuál estos menores se desarrollan a la par con otros menores en la misma condición que ellos, así como ejemplificar que procedimientos se realizaron durante la experiencia en búsqueda de recavar información que se pueda utilizar para lograr su bienestar, y analizar las condiciones de donde los menores provienen, para poder generar recomendaciones que permitan que los menores se desarrollen de forma adecuada y puedan mejorar su calidad de vida. 


\section{CAPÍTULO I: IDENTIFICACIÓN DEL PROBLEMA}

En la actualidad existe un gran índice de menores que son víctimas de abandono en el Perú. Esta situación es un problema social que afecta a muchas familias y principalmente a los menores que se encuentran expuestos a distintos factores de riesgo que los pueden llevar a ser víctimas de abandono. Este fenómeno social se encuentra estrechamente relacionado con aspectos como el maltrato infantil, la violencia familiar, el bajo nivel de educación de los padres, el bajo nivel económico de las familias involucradas en este fenómeno, el consumo de alcohol o drogas por parte de los progenitores o cuidadores, entre otros tipos de elementos.

Estos factores son las principales causas que influyen y determinan que los menores sean víctimas de abandono. En el caso del maltrato infantil, se hace referencia a los comportamientos diversos en los que los progenitores, apoderados, o personas mayores a cargo de los menores incurren, y que involucran un riesgo. Los comportamientos pueden ser intencionados o no, asi como pueden ser actos que omiten responsabilidad de su parte (Junco, 2014).

Asimismo, los casos de violencia familiar en el Perú, son múltiples y frecuentes. En el año 2014, se efectuaron más de 11,000 denuncias de violencia familiar a nivel nacional, contra los padres de familia o apoderados de los menores afectados en los hechos suscitados, y en donde el $57 \%$ de las víctimas eran mujeres y el $43 \%$ varones (Garmendía, 2015). Es importante resaltar que el tipo de violencia descrita en estos sucesos no sólo era física, sino también psicológica.

En el mismo plano, la violencia física hace referencia a la serie de acciones intencionadas que provoque lesiones físicas, mientras que la violencia psicológica denota un rango distinto de acciones, dentro de las cuáles están comprendidas el lenguaje violento hacia el menor, acciones que demuestren desprecio y poca valorización del menor, amenazas, manipulación, 
falta de afecto y vinculo familiar, ridiculización, amedrentamiento, rechazo, entre otras acciones que generen un impacto negativo en el autoconcepto, la identidad y la autoestima del menor (Del Águila-Escobedo, 2015).

De la misma manera, otro factor con impacto perjudicial en este problema, es el bajo nivel socioeconómico de las familias en donde se suscita el problema de abandono. El límite de disposición económica genera una serie de dificultades, dentro de las cuáles se encuentra, en la mayoría de casos, la imposibilidad de acceder a una educación adecuada, que genere integración social para las familias, así como un sentimiento de capacidad de elección en la búsqueda de una mejor calidad de vida. Es decir, las opciones de desarrollo disponibles para estas familias son escasas, debido a que su nivel socioeconómico los restringe a tratar de sobrevivir, dejando de lado aspectos vitales como la educación o, incluso, una alimentación adecuada. Las familias con bajos recursos presentan un alto nivel de incidencia de abandono de menores históricamente, debido a que en muchas ocasiones no pueden pagar los gastos que representan criar un hijo (Herrera, 2018). Es relevante traer a consideración que, según la Encuesta Nacional de Hogares (ENAHO), el índice de pobreza en el Perú, alcanzó el 21.7\% en el año 2017 (Instituto Nacional de Estadística e Informática [INEI], 2017).

En cuanto al consumo de alcohol o drogas, se puede mencionar que este funciona como un catalizador que genera una predisposición a que se cometan actos violentos por parte del consumidor debido al nivel de desinhibición y el descontrol de impulsos que el consumo provoca (Esbec y Echeburúa, 2016). Por un lado existe el riesgo que a partir del consumo, los menores puedan ser víctimas de abuso o maltrato de parte del consumidor, mientras que por otro lado, existe una tendencia a incurrir en un estado de dependencia y desfasamiento de la responsabilidad de los hijos por parte de los consumidores, por lo que existe el riesgo que estos puedan ser abandonados o ser dejados sin cuidado alguno. 
A finales del año 2015, la cantidad de menores identificados como víctimas de abandono en el Perú era aproximadamente de 19,865. Estos menores se encontraban bajo el cuidado de distintas instituciones del estado, encargadas de brindar protección, alimentación, estadía y en algunos casos educación a los menores (Herrera, 2018). Estas instituciones se encuentran reguladas por el gobierno del Perú. Asimismo, funcionan como espacios de protección y apoyo, donde los menores son llevados tras la comprobación del estado de abandono del que son víctimas.

El centro de atención residencial en el cuál se llevó a cabo la experiencia de trabajo sobre la que este reporte hace referencia, alberga a menores víctimas de abandono, desde su fundación en el año 1930. Este albergue consta de tres unidades residenciales: el hogar de mujeres, el hogar de varones y el hogar de infantes. Actualmente alberga a más de 250 menores. Tiene como objetivo principal cubrir las necesidades elementales de los menores en estado de abandono, como la salud, alimentación, vestido y educación de estos.

Dentro de la experiencia realizada en esta institución se tuvo como grupo de trabajo asignado a 16 menores de sexo masculino, con edades entre 12 y 16 años, en condición de abandono, del hogar de varones, desde la posición de interno de psicología entre el mes de Agosto del 2015 y Agosto del 2016. Este grupo provenía en su totalidad, de familias disfuncionales con pocos recursos. Todos los menores tenían al menos 7 años de residencia en la institución. A la par, 10 de ellos contaban con al menos uno de los progenitores, con un historial de consumo de drogas o alcohol $\mathrm{y}, 4$ de este grupo tenían al menos uno de los padres cumpliendo una condena efectiva en prisión. Finalmente, otro aspecto importante a considerar en los antecedentes contextuales con los que estos menores contaban, era la ausencia de al menos un progenitor debido al fallecimiento de este. Eran 2 los menores que tenían al menos uno de los padres en condición de fallecido. En ambos casos los decesos se dieron por abuso en el consumo de drogas. La mayoría de ellos presentaba dificultades para 
integrarse dentro de su hogar de convivencia y principalmente, evidenciaban dificultades para comunicarse y relacionarse con sus cuidadores y tutores, mostrando actitudes desafiantes o incurriendo en actos de indisciplina. Igualmente, se pudo identificar el uso de lenguaje violento y coprolálico. Los menores estudiaban en un colegio estatal en el horario de la tarde ubicado a 2 cuadras de la institución, para lo que se trasladaban todos los días con sus auxiliares, y regresaban por su cuenta.

Es así que se puede identificar a modo general, como parte de las necesidades de este grupo de menores en estado de abandono: un lugar de residencia, alimentación, vestimenta, afecto y educación. En el mismo sentido, pero bajo una perspectiva más detallada y definida a partir de sus característica particulares como población, estos menores necesitaban contar con modelos positivos de personas, reintegración social, diversidad de tareas y actividades integradoras, y ser educados en un sentido de responsabilidad y disciplina dentro de la institución que los cobijaba en dicho contexto. 


\section{CAPÍTULO II: DESCRIPCIÓN DE LAS ACTIVIDADES Y TAREAS REALIZADAS}

Dentro de la experiencia de trabajo llevada a cabo en la institución señalada previamente, las funciones y actividades se encontraban asignadas y delegadas por el área de Psicología. Cada interno que trabajaba en este área, tenía asignado un grupo de menores con los cuáles debía llevar a cabo las funciones asignadas. La distribución de los menores y los respectivos internos responsables de ellos, se realizaba bajo el criterio de la coordinadora del área de Psicología.

En las primeras semanas de interacción con los menores, previo a la realización de las funciones y actividades, los internos eran presentados ante ellos, para luego pasar a coordinar horarios disponibles de los menores, con los auxiliares responsables, y poder establecer un nuevo vínculo social y desarrollar rapport con los mismos, por medio de visitas a los eventos del hogar, conversaciones con cada uno de ellos, interacción en sus horas de alimentación, y presencia constante durante el día, en el hogar donde residían. Todo esto se llevaba a cabo para realizar las actividades y tareas establecidas con cada menor a cargo de cada interno.

A continuación se describirá y detallará las actividades y tareas realizadas durante la experiencia de trabajo con los 16 menores albergados en el centro de atención residencial señalado líneas arriba.

\subsection{Seguimiento}

Dentro de las tareas que componen la actividad de seguimiento, se encuentran las visitas y conversaciones con los menores a cargo, dentro de las cuales, se consideraba como objetivo principal el establecimiento y reforzamiento de la confianza y el vínculo entre el menor y el interno, así como la integración del interno dentro del desenvolvimiento regular del menor en la institución. 


\subsubsection{Visitas al hogar}

Se realizaban visitas diarias al hogar donde los menores vivían para conocer el estado de sus espacios personales (dormitorios y casilleros), el cumplimiento de su rol de actividades en el hogar, y tener conocimiento por medio del libro de incidencias y del reporte de los auxiliares, acerca de la conducta de los menores durante la semana. De la misma manera, estas visitas servían para identificar el interés y motivación de los menores, al realizar sus actividades académicas y otras responsabilidades personales.

\subsubsection{Visitas a eventos deportivos}

El hogar de Tomás Valle organizaba eventos deportivos y artísticos todos los meses, buscando la integración y participación de todos los menores que residían en dicha unidad. Se realizaban visitas y acompañamiento a los menores a lo largo de su participación en dichos eventos. Por medio de esta tarea se podía apreciar su interés en los deportes y actuaciones, así como su motivación y confianza en sí mismos.

\subsubsection{Seguimiento en horario de alimentación}

Se realizaban visitas en los horarios de alimentación, principalmente durante las horas de desayuno y almuerzo, y de forma esporádica durante la cena de los menores, para poder observar la conducta de los menores en un contexto donde compartían espacio y alimento con el hogar de varones en su totalidad. De la misma forma, se conversaba con los auxiliares para conocer si los menores asignados a la limpieza en el comedor, cumplían con sus tareas asignadas de forma adecuada. Estos momentos se utilizaban para coordinar reuniones o sesiones de conversación o con los menores y los cuidadores. 


\subsection{Evaluación}

\subsubsection{Aplicación de las pruebas}

La batería de pruebas utilizadas para la evaluación de los menores constaba un grupo de 4 pruebas específicas: el Test del Dibujo de la Figura Humana y el Test de la Familia, por medio de los cuáles se evaluaba el estado emocional actual del menor, el Test de Retención Visual de Benton, con el que se medía la memoria visual y las habilidades visoconstructivas, y la Prueba de Escala de Inteligencia WISC-III, a través de la cuál se estimaba la capacidad cognitiva e intelectual del menor. Esta última prueba se aplicaba en su tercera actualización debido a que era la versión que se encontraba validada en el Perú y permitía la evaluación de menores entre los 6 años y 16 años y 11 meses de edad. La evaluación se llevaba a cabo en horarios establecidos y coordinados con los auxiliares encargados de los menores, y en espacios preparados por el área de Psicología.

\subsubsection{Elaboración de informes}

Tras la realización de la evaluación, se recopilaban los resultados obtenidos para consolidar el perfil de cada menor con los diferentes aspectos evaluados como el área emocional, la maduración visomotora, el área cognitiva y la capacidad intelectual. Se realizaba la interpretación de resultados, y se elaboraba el reporte denominado "Informe Evolutivo", en donde se explicaban los resultados. En caso hubiese un ingreso nuevo de un menor en la institución, se realizaba la evaluación y luego se elaboraba el denominado "Informe Inicial". Estos informe pasaba posteriormente a consolidarse en el expediente del menor, junto con los documentos legales correspondientes. 


\subsection{Atención Terapéutica}

\subsubsection{Arteterapia}

Se realizaban sesiones individuales con los menores en donde se contaba con material didáctico como colores, plumones, temperas y óleos para pintar sobre papel y tela. Estas sesiones involucraban temas libres, en donde los menores podían desarrollar su creatividad y plasmar sus habilidades artísticas. Se llevaron a cabo al menos 4 sesiones de arteterapia con cada menor. Asimismo, este medio era un agente motivador para su autoestima y una forma de fortalecer el rapport con los menores, dado que consideraban la actividad como algo entretenido e interesante. Esta herramienta terapéutica permitía manejar su ansiedad y su salud mental, física y emocional (Cobo, 2018).

\subsubsection{Músicoterapia}

Se llevaron a cabo sesiones de musicoterapia en grupo de 3 menores por sesión una vez por mes. Para esto se tomó en cuenta el nivel de conocimiento musical y experiencia del interno a cargo para poder implementar sesiones con los menores. Se dispuso de instrumentos musicales de cuerda, proveidos por el interno, y percusivos con la intención de permitir que los menores expresen su creatividad por medio de ellos bajo la guía del interno, para tratar de componer una idea musical colectiva. Los menores integraban sus ideas y trabajaban en equipo en desarrollar una creación musical en conjunto. Canalizar la actividad musical de forma terapéutica permite que los menores liberen su carga emocional por medio de su expresión singular y creatividad personal (Montánchez y Orellana, 2015). 


\subsubsection{Psicoeducación}

Se realizaron sesiones grupales en donde se discutían y compartían temas importantes para los menores. Se les pedía que postulen temas que ellos consideraban como relevantes, y así se definían temas de conversación desde una perspectiva psicoeducativa. Algunos de los temas de estás conversaciones fueron el desarrollo de habilidades sociales, la comunicación asertiva, el rol personal, las relaciones de pareja adolescentes, el rol paterno, entre otros temas. Por medio de esto, se buscaba que se incremente la confianza entre los internos y el grupo de menores con el que se trabajaba, y principalmente que los menores incorporen estás conversaciones y conocimientos como parte de su entendimiento y autoconcepto. (Ramos-Díaz, Rodríguez-Fernández, Fernandéz-Zabala, Revuelta y Zuazagoitía, 2016).

\subsection{Elaboración de charlas para auxiliares}

A la par, se diseñaban charlas para los cuidadores y auxiliares de los menores, dentro de las cuáles se describían y explicaban temas relevantes sobre de su relación con los menores. Para ello se realizaba una labor de promoción y difusión del evento en los diferentes murales de la institución y los hogares. Dentro de los temas desarrollados en estas exposiciones se encuentran la importancia del rol paterno, 10 criterios para educar personas valiosas, criterios para educar a un adolescente, comunicación asertiva, entre otros temas. 


\section{CAPÍTULO III: RESULTADOS DE LA INTERVENCIÓN}

Debido a la situación de abandono de la que eran víctimas, los menores no mostraban seguridad para trabajar y colaborar con los internos en un principio. Fue determinante establecer paulatinamente un vínculo de confianza que les permitió tener la convicción de manifestar su ideas, hacer preguntas, consultar y principalmente comprender que el trabajo realizado en la institución estaba orientado a su bienestar y a su desarrollo personal. En ocasiones la negativa para fijar horarios de evaluación con los auxiliares era una dificultad, así como la falta de materiales disponibles en el área de Psicología.

Se estableció un vínculo positivo con ellos que trascendió específicamente en la calidad de comunicación que los menores lograron aprender a interiorizar. En un principio, las visitas que se realizaban al hogar de residencia, a los eventos deportivos y en las horas de desayuno, almuerzo y cena, sirvieron para definir la presencia del interno como parte de su día a día, para posteriormente, poder entablar conversaciones con ellos y generar un rapport que se fortalecía con cada visita. Este aspecto fue vital para poder lograr las evaluaciones posteriores con los menores dado que inspiraba seguridad y confianza. Es así que, tras contar con un vinculo positivo con los menores, se pudo traer a conversación durante las visitas al hogar y los campeonatos, temas como el estilo de comunicación que utilizaban entre ellos, el cuál en principio era soez y confrontador, y que tras establecer conversaciones durante las visitas con cada uno de ellos, se logró modificar positivamente. Este cambio tomó aproximadamente 6 meses. La mayoría de los menores cambiaron su estilo de comunicación por uno más asertivo y calmado, y se pudo lograr que comprendan que un ambiente positivo contribuía para su bienestar. Se logró entablar un vínculo de confianza importante con al menos 14 adolescentes. Este aspecto se logró desarrollar y profundizar aún más con las sesiones y tareas realizadas como parte de la atención terapéutica. 
Por otro lado, en la actividad de evaluación, se logró evaluar a 15 de los menores a excepción de uno, que fue evaluado por la coordinadora del área a pedido de ella. Las evaluaciones con los menores se lograron completar en un periodo de 9 meses, dentro de los plazos establecidos. Para lograr cumplir ese objetivo, se tuvo que desarrollar un lazo de confianza con los menores y así contar con su disposición e interés para ser parte de las evaluaciones. El proceso de evaluación a los menores era determinante para explorar e identificar dificultades en el aprendizaje, aspectos conductuales, así como posibles inconvenientes que podrían suscitarse en el área escolar, social y personal. Asimismo, se logró elaborar los informes respectivos de cada menor y consolidar los expedientes con información actualizada sobre las capacidades intelectuales, el estado emocional y la maduración visomotora de cada uno de ellos.

Asimismo, los resultados obtenidos a partir de la atención terapéutica, fueron satisfactorios. La totalidad de los menores mostraron interés en participar en las sesiones de arteterapia y músicoterapia, principalmente debido a que representaba una actividad novedosa que involucraba el desarrollo de su creatividad y la expresión de sus ideas por medio de la pintura y la música. Estas sesiones permitían establecer conversaciones durante la actividad, sobre como se sentían los menores en el hogar, qué ideas tenían sobre su futuro, entre otras conversaciones sobre su estado emocional, así como fortalecer el vínculo social con el interno. La autoexpresión por medio de este tipo de terapias permiten explorar aspectos sobre la propia persona y su autoconciencia, así como conocer más sobre sus emociones y pensamientos, de forma que al ver sus creaciones en la sesión se refuerce el concepto personal (Cobo, 2018). De la misma manera, las charlas psicoeducativas realizadas sirvieron para promover cambios de conducta positivos, principalmente en el lenguaje utilizado, la conducta entre los menores y con sus cuidadores, y las habilidades sociales, como se señaló líneas arriba. También se lograron cambios en el orden y rol personal de los menores. Dentro 
de las charlas se les pedía que describan sus responsabilidades y tareas en el hogar y que definan si eran necesarias y si tenían un impacto importante en el orden y armonía del hogar. Esta organización de criterios determinados por ellos mismos, les permitió analizar el alcance e impacto de sus acciones del día a día y generar cambios.

De la misma manera, se llevaron a cabo charlas sobre relaciones de pareja en la adolescencia, las cuales fueron de mucho interés para los menores dado que tenían entre 12 y 16 años de edad. Unos 8 menores del grupo total de 16 señalaron que tenían enamoradas, con quiénes frecuentaban a su regreso del colegio donde estudiaban por las tardes, hacia el hogar. En estas conversaciones se les brindo guía acerca del respeto hacia la otra persona y el compromiso dentro de una relación con otra persona. Asimismo, ellos hicieron preguntas y consultas acercas del tema. Existe una tendencia en la mayoría de adolescentes actualmente a rechazar la violencia contra la mujer, y los estereotipos sexistas (De la Osa, Andrés y Gómez, 2013). Es a partir de ese medio que se trabajó los conceptos de tolerancia y respeto hacia la pareja, lo que tuvo un impacto positivo en los menores, quienes se mostraron agradecidos de finalmente poder entablar este tipo de discusión con personas que tenían su confianza y que estaban realmente comprometidas con su bienestar y desarrollo personal.

Finalmente, los auxiliares que fueron parte de las charlas brindadas por el área de psicología, brindaron feedback acerca de la efectividad del uso de lo explicado en las charlas en su día a día. Señalaron que pudieron entender las ideas y conceptos ejemplificados para poder hacer uso de ellos en su labor de cuidadores en la institución, siendo el cambio más notorio la mejora en la comunicación entre los menores y ellos, así como un desarrollo de vínculo más importante con los menores, basado en el respeto y la empatía por el rol que cada uno cumplía en el hogar. 


\section{CONCLUSIONES}

- A partir del establecimiento de un rapport adecuado con los menores se pudo generar por medio del seguimiento y las conversaciones constantes con ellos, lo que impactó principalmente en su lenguaje cotidiano, que pasó de ser retador a ser cordial y asertivo, con sus pares y con sus cuidadores.

- El involucramiento del interno en las actividades deportivas, sociales y cotidianas de los menores fue un aspecto determinante para poder establecer confianza entre ambas partes, lo que a la larga serviría para desarrollar satisfactoriamente las sesiones de terapia en arte, música y psicoeducación, así como evaluar a la totalidad de los menores asignados.

- El proceso de evaluación a los menores fue útil para tener mayor claridad del impacto que la condición de abandono y el contexto previo personal había tenido en el área emocional, cognitiva, perceptiva y social de ellos, en conjunto con las sesiones de arte.

- Las charlas dictadas a los menores tuvieron resultados positivos en los menores. Los conceptos y ejemplos desarrollados acerca de modelos positivos, tolerancia, respeto, relaciones interpersonales, valores y responsabilidad personal, fueron recibidos y puestos en práctica por la mayoría de los menores de forma adecuada.

- Asimismo, las charlas realizadas con los cuidadores y auxiliares, generaron un lazo de comunicación más fluida entre ellos y los internos, lo que permitió una mejor coordinación y un incremento de interés en participar en las charlas que el área de Psicología realizaba. 


\section{RECOMENDACIONES}

- La institución trata de generar actividades para los menores para que el día a día no sea repetitivo y tan metódico; sin embargo las actividades que se realizan podrían ser más variadas e involucrar otro tipo de temas, y no solo limitar las opciones al área deportiva. Se debería inculcar el desarrollo del arte o la ciencia para presentar nuevas áreas de interés a los menores.

- Los materiales de trabajo en la institución son bastante limitados, principalmente para los menores que pasan los 12 años. La mayoría de los recursos se destinan al área de infantes. Se necesita mejorar las condiciones para el área de Psicología en el trabajo con los menores en la etapa de adolescencia de la institución.

- Los internos deberían contar con capacitaciones específicas más profundas que sean útiles al intervenir con los menores que tienen a cargo. La planificación y organización en la institución podría ser más integral y no tan segmentada entre las diferentes áreas.

- Las charlas y capacitaciones a los cuidadores y auxiliares deberían ser programadas con anterioridad y estar orientadas al logro de resultados con los menores, principalmente en tratar de alcanzar mejoras en la conducta de estos.

- Los menores requieren apoyo social y modelos positivos en su desarrollo como personas. En muchos contextos dentro de la institución la disciplina era exigida de forma muy rigurosa. Los espacios de confianza entre los internos y los menores contribuían a generar respuestas positivas. Se debería considerar ampliar el rango de intervención de los internos y del área de Psicología para buscar resultados positivos. 


\section{REFERENCIAS}

Cobo Robayo, V. (2018). Influencia del Arteterapia en el autoconcepto de niños de 6-12 años. (Trabajo de titulación para optar el título profesional de Licenciado de Psicología). Universidad San Francisco de Quito.

De la Osa, Z., Andres, S., Pascual, I. (2013). Creencias adolescentes sobre la violencia de género. Sexismo en las relaciones entre adolescentes. EJIPHE European Journal of Investigation in Health, Psychology and Education, 3(3), 265-275. https://doi.org/10.30552/ejihpe.v3i3.51

Del Águila-Escobedo, A. (2015). Violence and stress during childhood: is our future in play?. Acta Médica Peruana,32(2), 71-83. Recuperado de http://www.scielo.org.pe/scielo.php?script=sci_arttext\&pid=S1728$59172015000200002 \& \operatorname{lng}=$ es\&tlng=en.

Esbec, E. y Echeburúa, E. (2016). Abuso de drogas y delincuencia: consideraciones paa una valoración forense integral. Adicciones, 28(1), 28-56. http://dx.doi.org/10.20882/addiciones.790.

Fernández M. (2014): Maltrato infantil: Un estudio empírico sobre variables psicopatológicas en menores tutelados (tesis doctoral, Universidad de Murcia, España). Recuperado de https://www.tdx.cat/handle/10803/146291

Garmendia, F. (2016). La violencia en el Perú 2015. Anales de la Facultad de Medicina, 77(2), 153-161. https://dx.doi.org/10.15381/anales.v77i2.11838

Herrera Campoblanco, J. (2018). Políticas públicas de protección de niños en estado de abandono: Perú 1990-2015 (tesis de maestría). Recuperado de http://repositorio.ucv.edu.pe/handle/UCV/16209

Instituto Nacional de Estadística e Informática (INEI) - Presidencia del Consejo de Ministros (PCM) - Dirección Técnica de Demografía e Indicadores Sociales (DTDIS) - INEI. 
(2017). Perú - Encuesta Nacional de Hogares sobre Condiciones de Vida y Pobreza 2017. Recuperado de

http://webinei.inei.gob.pe/anda_inei/index.php/ddibrowser/613/export/?format=pdf\&g enerate $=$ yes

Junco, J. (2014). Análisis psicosocial del maltrato infantil. Avances en Psicología: Revista de la Facultad de Psicología y Humanidades. 22(2), 179-191. Recuperado de http://revistas.unife.edu.pe/index.php/avancesenpsicologia/article/view/188/173

Montánchez, M., Orellana, C. (2015). Aprendizaje socioemocional en la adolescencia a través de la musicoterapia. Iberoamérica Social: revista-red de estudios sociales, 4 , 164-174. Recuperado de http://iberoamericasocial.com/aprendizaje-socioemocionalen-la-adolescencia-a-traves-de-la-musicoterapia

Ramos-Díaz, E., Rodríguez-Fernández, A., Fernández-Zabala, A., Revuelta, L., \& Zuazagoitía, A. (2016). Apoyo social percibido, autoconcepto e implicación escolar de estudiantes adolescentes. Revista de Psicodidáctica, 21(2). Recuperado de http://www.ehu.eus/ojs/index.php/psicodidactica/article/view/14848/14228 
APÉNDICES 


\section{APÉNDICE 1: MODELO DE INFORME INICIAL}

INFORME PSICOLÓGICO INICIAL NXXX JSPSI-PPA 2015

\section{DATOS DE FILIACIÓN}

Nombres y Apellidos: $X X X X X X X X X$, Luis

Fecha de Nacimiento: 10/12/2002

Edad : 13 años 5 días

Fecha de Ingreso : :27/11/2015

Fecha Actual $\quad: 16 / 12 / 2015$

\section{MOTIVO DE INGRESO}

El menor ingresa el día 27 de noviembre del 2015, con oficio №̛X-2015.MIMPDGNNA-DIT.LJRL, con motivo basado en la investigación realizada por el Equipo Itinerante de la Dirección de Investigación Tutelar con ficha de atención №602-2015, proceso referido en el oficio citado previamente, el cual concluye que el menor no cuenta con un soporte familiar adecuado en la actualidad. El menor es producto de una presunta violación sexual incestuosa, por parte de su padre, $X X X X$ (51) y la hija de este señor, XXXX. El menor se encontraba viviendo con ambos padres, hasta los 11 años de edad, pero paso a quedarse solo bajo el cuidado del padre dado que ambos progenitores discutían con mucha frecuencia. A su vez, el señor XXXX señala que la madre del menor le fue infiel con el señor XXXX. El menor describe a su madre como una persona que lo maltrataba física y psicológicamente.

\section{RESULTADOS DE LA EVALUACIÓN PSICOLÓGICA}

XXX, obtiene un Cociente Intelectual que lo ubica dentro de la categoría de Normal Inferior. Con una Edad Mental de 10 años 7 meses, encontrándose un retraso de 2 años y 5 meses respecto a su edad cronológica.

El menor cuenta un mejor desempeño en el área Verbal, en la cual se mantiene en un nivel Promedio Inferior, mientras que en el área Ejecutiva, se encuentra en el nivel Fronterizo.

El área de mejor desempeño es la verbal, en la cual presenta habilidades para asociación de conceptos y, logra resolver problemas que se presentan en la vida cotidiana y en donde debe hacer uso de su juicio sentido común. Sin embargo, su desempeño decae cuando debe evocar conocimientos previos. En cuanto a su vocabulario es limitado para su edad, presentando dificultades para el manejo de palabras.

En el área de Velocidad de Procesamiento, presenta destreza y capacidad para el manejo de símbolos así como rapidez de asociación.

Por otro lado, en el área de Organización Perceptual, logra mantener con dificultad la atención y concentración para identificar objetivos y discriminar estímulos. Presenta dificultades mayores en relación a su coordinación visomotora y su organización no perceptiva.

Asimismo, en el área de Atención y Concentración, logra resolver operaciones númericas con cierta dificultad. De la misma manera, presenta cierta dificultad en el uso 
de su agilidad mental. A nivel de su maduración visomotora, se encuentra de acuerdo a edad cronológica.

XXX evidencia tener un estilo de pensamiento rígido que lo lleva a ser perfeccionista en la realización de sus actividades. El adolescente se muestra colaborador y comunicativo, además de presentar una buena capacidad de adaptación. Asimismo, el menor es consciente de su situación familiar y de internamiento.

\section{CONCLUSIONES}

XXX, a nivel intelectual, obtiene un Cociente Intelectual que lo ubica dentro de la Categoría Fronterizo. Con una Edad Mental de 9 años 4 meses. A nivel de su maduración visomotora, se encuentra de acuerdo a edad cronológica. A nivel emocional presenta un problema emocional moderado, siendo las áreas afectadas la: familiar y afectiva; sin embargo cuenta con recursos internos para poder superar su problemática actual; ya que con adecuada capacidad de adaptación, comunicación.

\section{RECOMENDACIONES}

\section{A nivel de Salud:}

- Evaluación Pediátrica para descartar dificultades a este nivel y seguimiento posterior de su estado de salud.

\section{A nivel Psicológico:}

-Seguimiento de su evolución y adaptación a la institución, mientras dure su internamiento en la institución.

- Reevaluación dentro de 6 meses; para precisar sus verdaderos recursos a nivel intelectual y emocional.

\section{A nivel del CEI}

- Que continúe su escolaridad (1ero de Secundaria) dentro de un Centro Educativo de la Comunidad.

\section{A nivel Social}

-Seguimiento y visita domiciliaria del caso; para ampliación de datos; puesto que no se cuenta con ningún dato de la historia personal del menor al momento de su ingreso. De encontrar algún familiar citarlo en la institución para poder elaborar la anamnesis.

\section{A nivel Legal:}

-Seguimiento de la Investigación Tutelar ; para definir el tiempo de permanencia del menor dentro de la institución. Averiguar si se cuenta con familia extensa que pueda hacerse cargo del cuidado y crianza de Cristhofer, de no ser posible esta medida; que se tome en cuenta el pedido de los padrinos de su hermano menor : Srs. XXXX; de querer hacerse cargo de todos los hermanos XXXX; puesto que cuentan con los recursos para ello, además que Luis y sus hermanos ya los conocen. 


\title{
APÉNDICE 2: MODELO DE INFORME EVOLUTIVO
}

\author{
INFORME PSICOLÓGICO EVOLUTIVO N XXX XXX-XX 2016
}

I. DATOS DE FILIACIÓN

Nombres y Apellidos : XXX, JEAN POOL

Fecha de Nacimiento : 29 de Diciembre del 2002

Edad

: 13 años 7 meses

Fecha de Ingreso $\quad: 5$ de Octubre del 2010

Fecha Actual : 23 de Agosto del 2016

\section{MOTIVO DE INGRESO}

El menor ingresa al centro el 05 de octubre del 2010 con la Causal Social de Abandono Potencial a pedido de la DIT-INABIF, junto con su hermano XXX (15).El caso es tomado por una denuncia telefónica realizada por la señora $X X X$, quién comunica que la señora $X X X$, madre del niño, lo dejaba solo y retornaba al hogar bajo los efectos del alcohol a altas horas de la noche llegando a ser negligente y exponiendo a los niños a situacion de riesgo.

\section{RESULTADOS PSICOLÓGICOS}

A nivel Intelectual, el menor presenta un Cociente Intelectual que la ubica dentro de la categoría "Normal Inferior". Con una Edad Mental que bordea los 11 años, 10 meses, encontrándose 21 meses por debajo de su edad cronológica.

En el área Verbal se ubica dentro de la categoría "Normal Inferior" mientras que en el área Ejecutiva, se encuentra dentro de la categoría "Normal Promedio".

En el análisis intra-test, el área de mejor desempeño es la de Velocidad de Procesamiento, pues presenta habilidades para mantener el ínterés en una tarea mecánica asi como emplear adecuadamente su atención para procesar información visual y perceptualmente. Al mismo tiempo presenta el mismo nivel de desempeño en el área de Organización Perceptiva, en donde muestra habilidades para interpretar material visual dentro de contextos donde existe tiempo limitado asi como relacionar estímulos visuales.

El área de menor desempeño es el de Ausencia de Distractibilidad, ya que la capacidad que tiene para mantenerse concentrado y atento es baja asi como la habiliidad para el cálculo númerico y la capacidad de retener a corto plazo estímulos con la memoria.

A nivel de Maduración Visomotora, obtiene un nivel adecuado para su edad cronológica.

Con respecto al nivel socioemocional, XXXX presenta ansiedad, retraimiento, inhibición de impulsos, inseguridad en si mismo asi como sentimientos de culpa e inadecuación al ambiente.

En el área de relaciones interpersonales, el menor se relaciona adecuadamente, principalmente con personas de su edad. En el caso de vincularse con personas mayores, muestra desconfianza y preocupación, asi como actitudes desafiantes ante la autoridad.

En el área familiar, el menor se siente identificado con su núcleo familiar y quiere ver a sus padres asi como el pertenecer a esa dinámica, sin embargo, presenta sentimientos de culpabilidad en relación a por qué el se encuentra albergado en la institución.

A nivel sexual, está identificado con su rol de género y sexo. Manifiesta interés y preocupación en esta área. 


\section{SITUACIÓN ACTUAL}

XXXX se encuentra ubicado en del Hogar de Varones. En relación a su salud, las auxiliares a cargo señalan que se encuenta estable. Con respecto a su apetito, mencionan que se alimenta adecuadamente. Por otro lado a veces es castigado por las auxiliar y como respuesta a esto el menor decide no participar del almuerzo. En relación a su sueño, señalan que duerme de forma tranquila.

Este año se encuentraba ubicado en 1er grado de secundaria, sección "C" en el Colegio Francisco Bolognesi hasta el mes de Julio. Posteriormente el menor se ha resistido al asistir al colegio alegando que no tiene deseos de estudiar ni de realizar labores academicas de ninguna índole. En la actualidad el menor se queda en el hogar realizando labores de limpieza y ayuda a las auxiliares. Se ha optado por buscar un programa de CETPRO para que el menor pueda realizar actividades de desarrollo en su favor. Sin embargo, no se ha logrado localizar un centro que dicte el curso de informática que el menor quiere realizar.

\section{RECOMENDACIONES}

- Se recomienda a la DIT-MIMP, definir la situación socio-legal del menor y de su dado el tiempo que lleva institucionalizado sin recibir visitas formales por parte de familiares.

- Se recomienda continuar brindando apoyo y seguimiento psicológico durante su presencia en la institución.

- Se recomienda mantener el seguimiento de la conducta, desempeño escolar y el desarrollo interpersonal del menor. 


\section{APÉNDICE 3: CUADRO DE PUNTAJES DEL ÁREA EMOCIONAL \\ Puntaje de Pruebas Proyectivas}

\begin{tabular}{|c|c|c|c|}
\hline Problemas & Leve (1 punto) & Moderado (2 puntos) & Severo \\
\hline $\begin{array}{c}\text { Relación } \\
\text { Interpersonal }\end{array}$ & $\begin{array}{l}\text { Timidez, inhibición, desconfianza; } \\
\text { pero logra superarlos }\end{array}$ & $\begin{array}{l}\text { Timidez, desconfianza e inhibición } \\
\text { que no logra superar con facilidad.. } \\
\text { Se relaciona mejor con pares que } \\
\text { con adultos (o viceversa). }\end{array}$ & $\begin{array}{l}\text { Problemas de } \\
\text { contacto } \\
\text { social. }\end{array}$ \\
\hline Conducta & $\begin{array}{l}\text { Negativismo, oposición, dificultad } \\
\text { inicial para aceptar órdenes o } \\
\text { adecuarse a límites. }\end{array}$ & $\begin{array}{l}\text { Mucha dificultad para aceptar } \\
\text { límites y respetar el encuadre de la } \\
\text { evaluación. Antecedentes de } \\
\text { problemas de conducta en el lugar } \\
\text { en que vivió anteriormente. }\end{array}$ & $\begin{array}{l}\text { Agresión } \\
\text { descontrolada, } \\
\text { falta de } \\
\text { límites, } \\
\text { conducta } \\
\text { social irregular } \\
\text { (robos } \\
\text { reiterados y } \\
\text { fugas a } \\
\text { conductas } \\
\text { delictivas). }\end{array}$ \\
\hline Autoestima & $\begin{array}{l}\text { Inseguridad, necesidad de } \\
\text { aprobación, actitudes de } \\
\text { sobrecompensación, poca valoración } \\
\text { de sí mismo que no afecta } \\
\text { significativamente su desempeño en } \\
\text { el medio. }\end{array}$ & $\begin{array}{l}\text { Afectan su desempeño, se hallan } \\
\text { indicadores significativos en la } \\
\text { prueba. }\end{array}$ & \\
\hline Afectivos & $\begin{array}{l}\text { Necesidades afectivas insatisfechas } \\
\text { y/o dificultad de expresión de afecto } \\
\text { sin que afecten significativamente su } \\
\text { desempeño en el medio. Inmadurez } \\
\text { emocional. }\end{array}$ & $\begin{array}{l}\text { Necesidades afectivas insatisfechas } \\
\text { y/o dificultad de expresión de } \\
\text { afecto que afectan su relación con } \\
\text { el medio y se hallan indicadores en } \\
\text { la prueba. }\end{array}$ & $\begin{array}{l}\text { Incapacidad } \\
\text { para } \\
\text { establecer } \\
\text { vínculos. }\end{array}$ \\
\hline $\begin{array}{l}\text { Dificultad de } \\
\text { Aceptación de } \\
\text { su Rol Sexual }\end{array}$ & $\begin{array}{l}\text { Figuras aparecen poco diferenciadas } \\
\text { de acuerdo con su edad. }\end{array}$ & $\begin{array}{l}\text { Problemas de identidad, confusión } \\
\text { de roles y/o dificultad de } \\
\text { aceptación de roles. }\end{array}$ & $\begin{array}{l}\text { Perversiones } \\
\text { sexuales, } \\
\text { prostitución. }\end{array}$ \\
\hline
\end{tabular}

\section{Puntaje de Pruebas Proyectivas}

\begin{tabular}{|cl|}
\hline \multicolumn{1}{|c|}{ Problemas } & \multicolumn{1}{c|}{ Severo } \\
\hline Cuadros Psicopatológicos & $\begin{array}{l}\text { Fobias y/o depresión severa. Desorganización } \\
\text { psicótica, psicopatía. }\end{array}$ \\
\hline
\end{tabular}

\begin{tabular}{|cll|}
\hline Problemas & \multicolumn{1}{c|}{ Leve y Moderado } & \multicolumn{1}{c|}{ Severo } \\
\hline Adaptación a Situaciones & Observación durante la & El niño(a) tiene características físicas \\
Nuevas & evaluación y/o referido en la & y de personalidad que, unido a \\
& anamnesis. & $\begin{array}{l}\text { indicadores emocionales en la } \\
\text { prueba, impedirán su adaptación al } \\
\end{array}$ \\
& & PPA. \\
\hline
\end{tabular}


Problemas

Trastornos de los Hábitos
Moderado (2 puntos)

Enuresis, tics.
Severo

Encopresis, tricotilomanía 


\section{APÉNDICE 4: MODELO DE REPORTE MENSUAL DE INTERNOS}

INFORME MENSUAL DE ACTIVIDADES DE LOS INTERNOS DE PSICOLOGÍA

PSICÓLOGO(A):

HOGAR:.

MES:

AÑO:

I. EVALUACIONES DIAGNÓSTICAS:

1.1 EXÁMENES DE INGRESO

\begin{tabular}{|l|c|c|c|c|}
\hline \multirow{2}{*}{ NOMBRE DEL MENOR } & \multirow{2}{*}{ EDAD } & \multicolumn{3}{|c|}{ RESULTADOS DE LA EVALUACIÓN } \\
\cline { 3 - 4 } & & \multicolumn{2}{|c|}{ APROBADO } & DESAPROBADO \\
\cline { 3 - 4 } & & REGULAR & CONDIC. & \\
\hline & & & & \\
\hline & & & & \\
\hline
\end{tabular}

1.2 EVALUACIONES PSICODIAGNÓSTICAS

1.2.1 INDIVIDUALES

1.2.1.1 DE ALUMNOS

\begin{tabular}{|l|l|l|l|l|l|}
\hline NOMBRE DEL MENOR & \multirow{2}{*}{ EDAD } & \multicolumn{4}{|c|}{ MOTIVO DE EVALUACIÓN } \\
\cline { 3 - 6 } & & REVALUACIÓN & P.APRENDIZAJE & P.COND. & JUZGADO/DIT \\
\hline & & & & & \\
\hline & & & & & \\
\hline
\end{tabular}

1.2.1.2 DE AULA

\begin{tabular}{|l|c|c|c|c|c|}
\hline AULA EVALUADA & \multirow{2}{*}{$\mathbf{N}^{\circ}$} & \multicolumn{4}{|c|}{ MOTIVO DE EVALUACIÓN } \\
\cline { 4 - 6 } & ALUMNOS & APLICACIÓN & CALIFICACIÓN & INTERPRETACIÓN & INFORME \\
\hline & & & & & \\
\hline & & & & & \\
\hline
\end{tabular}

II. ATENCIÓN TERAPÉUTICA

2.1 INDIVIDUAL

2.1.1 PSICOTERAPIA

\begin{tabular}{|c|c|c|}
\hline NOMBRE DEL MENOR & EDAD & $\mathbf{N}^{\circ}$ DE SESIONES REALIZADAS \\
\hline & & \\
\hline & & \\
\hline
\end{tabular}


2.1.2 CONSEJO Y APOYO PSICOLÓGICO

\begin{tabular}{|l|l|l|}
\hline NOMBRE DEL MENOR & EDAD & $\mathbf{N}^{\circ}$ DE SESIONES REALIZADAS \\
\hline & & \\
\hline & & \\
\hline & & \\
\hline & & \\
\hline
\end{tabular}

2.2 GRUPAL

\begin{tabular}{|l|c|c|c|c|c|}
\hline $\mathbf{N}^{\circ}$ DE MENORES ATENDIDOS & \multirow{2}{*}{ GDO. ESCOLAR } & \multicolumn{4}{|c|}{ TIPO DE GRUPO } \\
\cline { 3 - 6 } & & TER. & CREAT. & E.COG & OPER. \\
\hline & & & & & \\
\hline & & & & & \\
\hline
\end{tabular}

III. SEGUIMIENTOS Y TUTORIAS

\begin{tabular}{|l|l|l|l|l|l|l|}
\hline NOMBRE DEL MENOR & \multirow{2}{*}{ EDAD } & \multicolumn{3}{|c|}{ ENTREV. REALIZADAS } & OTRAS \\
\cline { 3 - 6 } & & MEN. & HOG. & C.E. & APOD. & ACCIONES \\
\hline & & & & & & \\
\hline & & & & & & \\
\hline & & & & & & \\
\hline & & & & & & \\
\hline & & & & & & \\
\hline & & & & & & \\
\hline & & & & & & \\
\hline
\end{tabular}

IV. PROGRAMAS ESPECÍFICOS: (Acciones en los Programas de Estimulación Temprana, Orientación Sexual, Orientación Vocacional, Etc.)

V. ASESORÍA Y APOYO TECNOLÓGICO

5.1 A NIVEL DE PERSONAL

\begin{tabular}{|l|l|l|c|}
\hline NOMBRE & CARGO & MOTIVO & $\begin{array}{c}\mathbf{N}^{\circ} \\
\text { ENTREV. }\end{array}$ \\
\hline & & & \\
\hline & & & \\
\hline & & & \\
\hline & & & \\
\hline
\end{tabular}

5.2 A NIVEL DE PADRES O APODERADOS

Reuniones de asesoría (especificar las acciones realizadas y el objetivo)

VI. INVESTIGACIONES 
Especificar los datos o aspectos en que participó en el mes: marco teórico, aplicación de pruebas, calificación, interpretación, análisis de resultados, elaboración de informe final.

\section{SUPERVISIONES}

7.1 Reuniones Individuales de Supervisión:

7.2 Reuniones Grupales de Supervisión:

Aspectos Tratados

VIII. INFORMES ENTREGADOS:

IX. MATERIAL PREPARADO:

X. OTROS TRABAJOS REALIZADOS:

XI. BIBLIOGRAFÍA O MATERIAL REVISADO:

XII. APRECIACIÓN DE SU TRABAJO EN EL MES:

$\begin{array}{llll}\text { DEFICIENTE } & \text { REGULAR } & \text { BUENO } & \text { MUY BUENO }\end{array}$

FECHA:

FIRMA INTERNO (A)

FIRMA SUPERVISOR 


\title{
APÉNDICE 5: GUÍA DE ACTIVIDADES DE CHARLA DE ORIENTACIÓN
}

\author{
Charla de Orientación
}

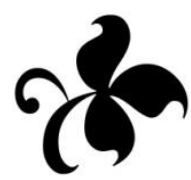

\section{Sesión 1: Relaciones de Pareja en Adolescentes}

Objetivo: Informar a los adolescentes sobre los distintos aspectos de una relación de pareja sana y responsable

\begin{tabular}{|c|c|c|}
\hline Actividad & Tarea/verbalización & Tiempo \\
\hline $\begin{array}{l}\text { Presentación de la charla } \\
\text { y el objetivo }\end{array}$ & $\begin{array}{l}\text { Se presenta el nombre del taller, } \\
\text { mencionando la realidad y la etapa de } \\
\text { desarrollo por la que están pasando. } \\
\text { Posteriormente se menciona el objetivo } \\
\text { del taller y los temas a tratar }\end{array}$ & 4 minutos \\
\hline $\begin{array}{l}\text { Participación libre con } \\
\text { pregunta rompe hielo de } \\
\text { iniciación }\end{array}$ & $\begin{array}{l}\text { “¿Qué es para ustedes una relación de } \\
\text { pareja?” } \\
\text { Se espera que respondan } \\
\text { indiscriminadamente de si tienen o no } \\
\text { pareja }\end{array}$ & 10 minutos \\
\hline Reflexión 1 & $\begin{array}{l}\text { "¿Y han pensado alguna vez que la } \\
\text { relación en pareja también incluye } \\
\text { desarrollo, crecimiento mutuo y } \\
\text { responsabilidad?" }\end{array}$ & 5 minutos \\
\hline Reflexión 2 & $\begin{array}{l}\text { “¿Qué valores creen que son los } \\
\text { necesarios en la relación para alcanzar } \\
\text { los aspectos anteriores? }\end{array}$ & 10 minutos \\
\hline Pregunta situacional & $\begin{array}{l}\text { “¿Qué harían en una situación de } \\
\text { discordancia con su pareja?” } \\
\text { *Por ejemplo, si tuviesen que decidir a } \\
\text { qué lugar ir a comer y no se ponen de } \\
\text { acuerdo, ¿Qué harían? } \\
\text { *Otro ejemplo, a ella no le gustan tus } \\
\text { amigos, pero tú quieres pasar tiempo } \\
\text { con ellos y con ella, de modo que le } \\
\text { propones estar todos juntos pero ella se } \\
\text { niega una y otra vez, ¿Qué harían? }\end{array}$ & 15 minutos \\
\hline Dinámica \#1 & $\begin{array}{l}\text { Participantes: todos } \\
\text { Materiales: Una Coca-Cola y papitas } \\
\text { Lays } \\
\text { Se forman dos grupos, en el primero } \\
\text { estarán Piero Joya y Diego Salazar, en el } \\
\text { segundo grupo están todos los demás. } \\
\text { El objetivo de la dinámica es que Piero } \\
\text { Joya intente llegar al premio sin } \\
\text { importar el método, mientras que todos }\end{array}$ & 10 minutos \\
\hline
\end{tabular}




\begin{tabular}{|c|c|c|}
\hline & $\begin{array}{l}\text { los demás intentarán impedírselo. } \\
\text { Al grupo grande se le especifica en } \\
\text { privado que deben impedir que Piero o } \\
\text { Diego lleguen al premio, excepto si } \\
\text { piden por favor, ya que solamente en } \\
\text { ese caso se les permitirá llegar al } \\
\text { premio. } \\
\text { Una vez que se cumpla el tiempo de } 4 \\
\text { minutos para Piero Joya, se detiene la } \\
\text { dinámica y se le permite jugar a Diego. }\end{array}$ & \\
\hline Reflexión 3 y cierre & $\begin{array}{l}\text { Se comenta sobre el estilo de } \\
\text { afrontamiento que tienen cada uno de } \\
\text { ellos al momento de resolver algún } \\
\text { problema y cómo eso les puede ayudar } \\
\text { a conseguir sus objetivos en la vida. } \\
\text { Asimismo se trata de que hagan insight } \\
\text { sobre si se les hubiese ocurrido ser } \\
\text { asertivos ante los problemas reales de } \\
\text { la vida cotidiana. } \\
\text { Se cierra la primera sesión }\end{array}$ & 8 minutos \\
\hline & & $\begin{array}{l}\text { Tiempo total: } 62 \\
\text { minutos }\end{array}$ \\
\hline
\end{tabular}

Int. Ps. Jorge Sifuentes Palomino 